\title{
BDNF - A PROBABLE COMMON PATHOGENETIC FACTOR FOR SCHIZOPHRENIA AND DEPRESSIVE EPISODE
}

\author{
Shishkov R. ${ }^{1}$, T. Chervenkov ${ }^{2}$ \\ ${ }^{1}$ Department of Psychiatry and Medical Psychology, Medical University of Varna, \\ ${ }^{2}$ Laboratory of Clinical Immunology, Medical University of Varna
}

\begin{abstract}
BDNF is a neurotrophic factor synthesized in the central nervous system (CNS) and the periphery. It passes through the brain-blood barrier. Peripheral BDNF can exert a trophic effect in the CNS and different plasma BDNF levels in schizophrenia, depression and bronchial asthma could contribute to negative correlation between these diseases. The aim of the present study was to assess peripheral BDNF levels in these diseases and to relate them to their estimated comorbidity. Plasma BDNF was assessed by immunoassay while disease incidence rate and comorbidity was assessed by database retrieval in patients suffering from schizophrenia, depressive episode and bronchial asthma. A lower plasma BDNF level was observed in patients with schizophrenia and depressive episode compared to those suffering from bronchial asthma and a negative comorbidity between diseases was registered. These observations support the important role of BDNF in the pathogenesis of schizophrenia and depression and can explain their negative comorbidity with bronchial asthma.
\end{abstract}

Key words: BDNF, schizophrenia, depressive episode, bronchial asthma, comorbidity

\section{INTRODUCTION}

There are studies and hypotheses which link the pathogenesis of schizophrenia with the concentration and activity of neural trophic factors like BDNF, NT-3, etc. . There is abundant evidence of the relationship between neurotrophins and schizophrenia. BDNF is, probably, of highest importance for the genesis of schizophrenia and its polymorphic variants. There is also a point of view which states that BDNF is not single factor but rather is interconnected with NT-3, i.e. neurotrophins have complex influence on the brain processes. There are similar observations concerning the affective disorders and depressive episodes with psychotic symptoms in the premises of bipolar affective disorder (BAD) and recurrent depressive disorder (RDD).

Evidence for BDNF involvement in the pathogenesis of schizophrenia comes from observations that its level is lower than that in healthy controls even before clinical presentation of the first disease episode. A lower BDNF level has been consistently observed during the disease course, too. It is considered that the deficiency of this major trophic factor disrupts an array of brain processes associated with neuronal plasticity, progenitor migration, apoptosis and mediator function. This mechanism is, probably, also valid

\footnotetext{
Address for correspondence:

R. Shishkov, Dept. of Psychiatry and Medical Psychology,

Medical University of Varna,

55 Marin Drinov Str., 9002 Varna, Bulgaria

e-mail: rshishkov@abv.bg
}

for depressive disorders considering the model for common genesis of these diseases.

Bronchial asthma is a disease associated with a higher BDNF level. There are observations that the level of this neurotrophic factor increases progressively during the period of chronic allergic inflammation . Neurotrophins are produced by epithelial cells in the lung and the airways and these have been considered as the 'big source of NGF and BDNF'.

The role of neurotrophins in bronchial asthma is related to their effects on the peripheral nervous system and the activity of cells of the immune system within the airways. BDNF is capable of passing through the blood-brain barrier and, in this way, the peripheral level would affect processes within the central nervous system (CNS).

Considering the dissociation of peripheral BDNF level in these diseases we aimed at studying the comorbidity of bronchial asthma and schizophrenia as well as of bronchial asthma and depressive episode and examining the level of BDNF in peripheral blood of bronchial asthma, schizophrenia and depressive episode.

\section{MATERIAL AND METHODS}

\section{Patients \\ Comorbidity of schizophrenia and bronchial asthma}

The study was conducted in the information database of St. Marina University Hospital of Varna during the period 
from 2002 till 2009. This is the only hospital in Varna region with specialized clinics of allergic and psychiatric diseases and thus it is expected that the majority of patients with these diseases are registered in the information database.

The records of 1891 schizophrenia patients were checked for comorbidity with bronchial asthma, those of 1591 bronchial asthma patients - for comorbidity with schizophrenia, and those of 2510 depressive syndrome patients - for comorbidity with bronchial asthma.

The psychiatric diseases were classified according to ICD-9 and ICD-10. In order to simplify the data analysis all the diagnostic codes were translated to ICD-10. The diagnostic codes according to ICD-10 were the following:

- patients with schizophrenia were classified as 'paranoid schizophrenia' (F20.0) according to ICD-10;

- patients with depressive syndrome were classified as 'recurrent depressive disorder' (F33), 'bipolar affective disorder' (F31), 'depressive episode - moderate severity depressive episode' (F32.1) and 'severe depressive episode with psychotic symptoms' (F32.3) were aggregated in 'depressive syndrome' according to ICD-10

- patients with bronchial asthma were classified as 'nonatopic bronchial asthma' (J45) according to ICD-10.

\section{$B D N F$ levels in blood plasma}

Blood plasma BDNF levels of were examined in the following groups:

1. a total of 31 patients with schizophrenia, 15 males at a mean age of 31 years (range 18-48 years) and 16 females at a mean age of 37,4 years (range, 18-48 years). At the moment of recruiting, the patients did not present with any acute or exacerbated chronic somatic disease. All of them were treated with standard antipsychotic therapy consisting of second-generation antipsychotic drugs. The patients were sampled for the period of disease relapse due to discontinuation of therapy and after at least 20 days of treatment and clinical remission;

2. ten female patients depressive syndrome at a mean age of 51,2 years (range, 38-60 years) were sampled for the period of disease relapse and clinical remission;

3. ten patients with bronchial asthma, 8 females at a mean age of 46,7 years (range, 29-73 years) and two males at a mean age of 32,7 years. The patients were sampled once for the subacute period and underwent a standard antiasthmatic therapy;

4. a control group consisting of 10 healthy volunteers, 4 males aged between 33 and 42 years and 6 females aged between 34 and 38 years.

\section{Methods}

Data were retrieved from the aforementioned information database for the period from 2002 till 2011 and statistically aggregated by its specialized software. Patients' records were accessed by patient's name and unique ID.
BDNF concentration (in $\mathrm{pg} / \mathrm{mL}$ ) was estimated in blood plasma. For separation of blood plasma EDTA anticoagulated blood was collected in vacuum closed collection system (Vacuette, Greiner Bio One). Blood was transported on ice and plasma was separated within 30 minutes after collection by centrifugation at $1000 \mathrm{~g}$ for 15 min. Blood plasma samples were stored at $-80^{\circ} \mathrm{C}$ until analyzed. Analysis of BDNF concentration was performed using Quantikine Human BDNF Immunoassay ELISA kit, catalog number DBD00 (R\&D Systems) according to manufacturer's instructions.

Statistical data processing was done by using the dispersion analysis (ANOVA), multiple comparison Mann-Whitney non-parametric test and percent distribution.

\section{RESULTS}

We established that only $4(0,25 \%)$ of 1591 patients with bronchial asthma presented with accompanying schizophrenia while only $4(0.21 \%)$ of 1891 with schizophrenia presented with accompanying bronchial asthma. These four patients were the same in both groups. Some 29 $(1,15 \%)$ of 2510 patients with depressive syndrome presented with accompanying bronchial asthma. It is evident that both comorbidities, i. e. of bronchial asthma and schizophrenia, on the one hand, and bronchial asthma and depressive syndrome, on the other hand, are lower than the expected incidence rate in Bulgaria. The results of the disease prevalence are shown in Table 1.

Table 1. Comorbidity of bronchial asthma (BA), schizophrenia (Sch) and recurrent depressive disorder

\begin{tabular}{|c|c|c|}
\hline $\begin{array}{l}\text { Comorbid } \\
\text { patients }\end{array}$ & $\begin{array}{c}\text { Number of patients } \\
\text { by disease }\end{array}$ & Comorbid frequency \\
\hline BA and Sch & $\mathrm{BA}$ & $\mathrm{BA}$ and $\mathrm{Sch} / \mathrm{BA} \%$ \\
\hline$n=4$ & $\mathrm{n}=1591$ & $0,25 \%$ \\
\hline BA and Sch & Sch & $\mathrm{BA}$ and $\mathrm{Sch} / \mathrm{Sch} \%$ \\
\hline$n=4$ & $\mathrm{n}=1891$ & $0,21 \%$ \\
\hline $\mathrm{BA}$ and RDD & RDD & BA-RDD / RDD\% \\
\hline $\mathrm{n}=29$ & $\mathrm{n}=2510$ & $1,15 \%$ \\
\hline $\mathrm{BA}$ and RDD & BA & BA-RDD / BA\% \\
\hline$n=29$ & $\mathrm{n}=1591$ & $1,82 \%$ \\
\hline
\end{tabular}

There are statistically significant difference in the variance between all the groups tested (ANOVA, $p<0,05$ ) and intergroup differences (Mann-Whitney test) in BDNF concentrations between: i) the patients with schizophrenia (in relapse) compared to those with bronchial asthma $(\mathrm{p}<0,05)$; ii) the patients with bronchial asthma compared to those with depressive syndrome (in relapse) $(p<0,05)$, and iii) the patients with depressive syndrome (in relapse) compared to the control group $(\mathrm{p}<0,01)$. 


\section{DISCUSSION}

The estimated comorbidity incidence rate of bronchial asthma and schizophrenia supports a previous study of ours showing an extremely low comorbidity between these two diseases (17). These results are contradictory to other ones in the literature available. A higher than expected comorbidity of bronchial asthma and schizophrenia was established. However, in their study bronchial asthma is included as a part of atopic diseases while we included the nonatopic infectious-allergic form of this disease.

We reveal a lower than expected comorbidity incidence of depressive syndrome and bronchial asthma, too. When interpreting this fact one should have in mind that many patients with bronchial asthma have depressive experiences because of psychogenic causes and antiasthmatic treatment. In our study, the patients with 'endogeneous depression' were included only.

Our results concerning the BDNF blood plasma levels are concordant with the literature available, e.g. about higher BDNF levels in bronchial asthma patients than in patients with schizophrenia and depression . Besides BDNF concentrations in the patients with bronchial asthma are higher than in the control group. These results are demonstrated on Fig. 1.

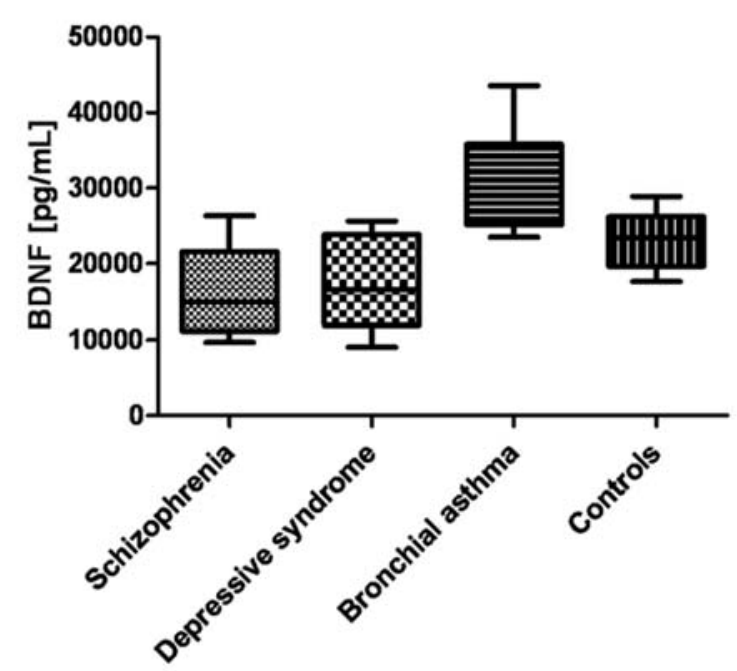

Fig 1. BDNF concentration (pg/mL) in schizophrenia, depressive syndrome, bronchial asthma and healthy controls

The outlined negative comorbidity of bronchial asthma and schizophrenia as well as of bronchial asthma and depression proved in epidemiological studies along with the marked differences in BDNF blood plasma levels in these diseases reflect, probably, one of the milestones of the pathogenesis of schizophrenia and depressive syndrome. This statement could be viewed in two aspects: on one hand, the lower BDNF concentration in the CNS could cause disturbed neuronal plasticity, progenitor migration and apoptosis, and on the other hand, by its concentration, BDNF could exert different effects due to its different poly- morphic variants. These variants would not have the effect of the wild type of BDNF. In this way, these aforementioned mechanisms of neuronal plasticity, apoptosis and progenitor migration, mainly in the regions associated with schizophrenia development could prove biologically ineffective and contribute to the development of schizophrenia. Similar arguments could be drawn to the depressive syndrome, i. e. depressive episode with psychotic symptoms and depressive episode in the premises of bipolar affective disorder and recurrent depressive disorder.

This predication correlates with the idea that the higher BDNF level in bronchial asthma could possess a neuroprotective effect in the CNS and prevent the development of schizophrenia in some cases. This is a possible explanation of the established lower than expected comorbidity of bronchial asthma and schizophrenia and of bronchial asthma and depressive syndrome, respectively. The similarity between the lower BDNF level in schizophrenia and depressive syndrome compared to healthy individuals is an indirect evidence of the existence of common mechanisms in the pathogenesis of schizophrenia and 'psychotic' depressive episode.

\section{REFERENCES}

1. Angelucci, F., S. Brene, A. A. Mathe. BDNF in schizophrenia, depression and corresponding animal models.- Mol. Psychiatry, 10, 2005, No 4, 345-352.

2. Chen, Y. H., H. C. Lee, H. C. Lin. Prevalence and risk of atopic disorders among schizophrenia patients: a nationwide population based study.Schizophr. Res., 108, 2009, No 1-3, 191-196.

3. Gorski, J. A., S. R. Zeiler, S. Tamowski, $\mathrm{K}$. R. Jones. Brain-derived neurotrophic factor is required for the maintenance of cortical dendrites.- $J$. Neurosci., 23, 2003, No 17, 6856-6865.

4. Green, M. J., S. L. Matheson, A. Shepherd, C. S. Weickert, V. J. Carr. Brain-derived neurotrophic factor levels in schizophrenia: a systematic review with meta-analysis.- Mol. Psychiatry, 16, 2011, No 9, 960-972.

5. Hahn, C., A. P. Islamian, H. Renz, W. A. Nockher. Airway epithelial cells produce neurotrophins and promote the survival of eosinophils during allergic airway inflammation.- J. Allergy Clin. Immunol., 117, 2006, No 4, 787-794.

6. Hattori, M., H. Kunugi, A. Akahane, H. Tanaka, S. Ishida, T. Hirose, et al. Novel polymorphisms in the promoter region of the neurotrophin-3 gene and their associations with schizophrenia.- Am. J. Med. Genet., 114, 2002, No 3, 304-309.

7. Jarskog, L. F. Apoptosis in schizophrenia: pathophysiologic and therapeutic considerations.Curr. Opin. Psychiatry, 19, 2006, No 3, 307-312.

8. Krebs, M. O., O. Guillin, M. C. Bourdell, J. C. Schwartz, J. P. Olie, M. F. Poirier, et al. Brain derived neurotrophic factor (BDNF) gene variants association with age at onset and therapeutic 
response in schizophrenia.- Mol. Psychiatry, 5, 2000, No 5, 558-562.

9. Lewin, G. R., Y. A. Barde. Physiology of the neurotrophins.- Ann. Rev. Neurosci., 19, 1996, 289-317.

10. Lu, B. BDNF and activity-dependent synaptic modulation.- Learn. Mem., 10, 2003, No 2, 86-98.

11. Martinowich, K., H. Manji, B. Lu. New insights into BDNF function in depression and anxiety.Nat. Neurosci., 10, 2007, No 9, 1089-1093.

12. Nassenstein, C., A. Braun, V. J. Erpenbeck, M. M. Lommatzsch, S. Schmidt, N. Krug, et al. The neurotrophins nerve growth factor, brain-derived neurotrophic factor, neurotrophin-3, and neurotrophin-4 are survival and activation factors for eosinophils in patients with allergic bronchial asthma.- J. Exp. Med., 198, 2003, No 3, 455-467.

13. Nikoletopoulou, V., H. Lickert, J. M. Frade, C. Rencurel, P. Giallonardo, L. Zhang, et al. Neurotrophin receptors TrkA and TrkC cause neuronal death whereas TrkB does not.Nature, 467, 2010, No 7311, 59-63.

14. Nockher, W. A., H. Renz. Neurotrophins and asthma: novel insight into neuroimmune interaction.J. Allergy Clin. Immunol., 117, 2006, No 1, 67-71.
15. Pan, W., W. A. Banks, M. B. Fasold, J. Bluth, A. J. Kastin. Transport of brain-derived neurotrophic factor across the blood-brain barrier.Neuropharmacology, 37, 1998, No 12, 1553-1561.

16. Renz, H., S. Kerzel, W. A. Nockher. The role of neurotrophins in bronchial asthma: contribution of the pan-neurotrophin receptor p75.- Progr. Brain Res., 146, 2004, 325-333.

17. Shishkov, R., E. Georgiev, K. Chernev. Relationships between some psychosomatic disorders and schizophrenic psychosis.- Izvestiya na Sayuza na uchenite-Varna, Seria medicina i biologiya, 2-2002/1-2003, 16-19 (in Bulgarian).

18. Szekeres, G., A. Juhasz, A. Rimanoczy, S. Keri, Z. Janka. The C270T polymorphism of the brain-derived neurotrophic factor gene is associated with schizophrenia.- Schizophr. Res., 65, 2003, No 1, $15-18$.

19. Xu, B., K. Zang, N. L. Ruff, Y. A. Zhang, S. K. McConnell, M. P. Stryker, et al. Cortical degeneration in the absence of neurotrophin signaling: dendritic retraction and neuronal loss after removal of the receptor TrkB.- Neuron, 26, 2000, No 1, 233-245. 